\title{
Quantifiable Minimal Residual Disease Positivity
}

National Cancer Institute

\section{Source}

National Cancer Institute. Quantifiable Minimal Residual Disease Positivity. NCI

Thesaurus. Code C124432.

Presence of quantifiable residual disease based on laboratory techniques. 